Pacific

Journal of

Mathematics

UNIT VECTOR FIELDS ON REAL SPACE FORMS

WHICH ARE HARMONIC MAPS

DOMENICO PERRONE

Volume 239 No. 1

January 2009 


\title{
UNIT VECTOR FIELDS ON REAL SPACE FORMS WHICH ARE HARMONIC MAPS
}

\author{
DOMENICO PERRONE
}

\begin{abstract}
In 1998, Han and Yim proved that the Hopf vector fields, namely, the unit Killing vector fields, are the unique unit vector fields on the unit sphere $S^{3}$ that define harmonic maps from $S^{3}$ to $\left(T^{1} S^{3}, \widetilde{G}_{s}\right)$, where $\widetilde{G}_{s}$ is the Sasaki metric. In this paper, by using a different method, we get an analogue of Han and Yim's theorem for a Riemannian three-manifold with constant sectional curvature $k \neq 0$. An immediate consequence is that there does not exist a unit vector field on the hyperbolic three-space that defines a harmonic map. We also extend this result for Riemannian $(2 n+1)$-manifolds $(M, g)$ of constant sectional curvature $k>0$ with $\pi_{1}(M) \neq 0$.
\end{abstract}

\section{Introduction}

The existence and explicit construction of harmonic mappings between two given Riemannian manifolds $(M, g)$ and $\left(M^{\prime}, g^{\prime}\right)$ are two of the most fundamental problems of the theory of harmonic mappings. If $M$ is compact and $M^{\prime}$ has nonpositive sectional curvature, then any smooth map from $M$ to $M^{\prime}$ can be deformed into a harmonic map using the heat flow method [Eells and Sampson 1964]. However, there is no general existence theory of harmonic mappings if the target manifold does not satisfy the nonpositivity curvature condition. This fact makes it interesting to find harmonic maps defined by vector fields and unit vector fields [Abbassi et al. 2007; 2008; Benyounes et al. 2007b; 2007a; Ishihara 1979; Nouhaud 1977; Perrone 2003; 2005; Rukimbira 2002; Tsukada and Vanhecke 2001].

Let $\mathfrak{X}^{1}(M)$ be the set of all smooth unit vector fields on $(M, g)$, which we suppose to be nonempty, or, equivalently, we suppose the Euler-Poincare characteristic of $M$ vanishes. Let $\left(T_{1} M, \widetilde{G}_{s}\right)$ be the unit tangent sphere bundle equipped with the Sasaki metric $\widetilde{G}_{s}$. A unit vector field $V \in \mathfrak{X}^{1}(M)$ determines a map between $(M, g)$ and $\left(T_{1} M, \widetilde{G}_{S}\right)$, and the energy of $V$ is defined as the energy of the corresponding map $V:(M, g) \rightarrow\left(T_{1} M, \widetilde{G}_{s}\right)$. Of course the first candidates in

MSC2000: 58E20, 53C43.

Keywords: harmonic maps, unit Killing vector fields, real space forms, Riemannian $g$-natural metrics.

Supported by funds of the MIUR and of the Universita del Salento. 
the study of the harmonicity of unit vector fields are the Killing vector fields: on the unit sphere $S^{2 m+1}$ equipped with the canonical metric, the unit Killing vector fields are exactly the Hopf vector fields; see [Wiegmink 1995]. Then, Han and Yim [1998] proved that a unit Killing vector field $\xi$ determines a harmonic map from $S^{2 m+1}$ to $\left(T_{1} S^{2 m+1}, \widetilde{G}_{s}\right)$. The same result holds if one considers a Riemannian manifold $(M, g)$ of constant sectional curvature $\kappa>0$. In dimension three they showed the following interesting result.

Theorem A [Han and Yim 1998]. The unit vector fields that define harmonic maps on the unit sphere $S^{3}$, with respect to the Sasaki metric $\widetilde{G}_{s}$, are precisely the Hopf vector fields or, equivalently, the unit Killing vector fields.

Recently, a very large family of metrics $G$ on $T M$, called Riemannian $g$-natural metrics, has been considered and studied [Abbassi and Sarih 2005; Abbassi and Calvaruso 2007]. This family of metrics, which includes the Sasaki metric $G_{s}$, the Cheeger-Gromol metric and other well-known Riemannian metrics on $T M$, depends on six arbitrary smooth real functions defined on $[0,+\infty)$ [Abbassi and Sarih 2005]. The restrictions $\widetilde{G}$ of such metrics to the tangent sphere bundle $T_{1} M$ possess a simpler form and globally depend on four real parameters $a, b, c$ and $d$ satisfying some inequalities (the parameters $a=1$ and $b=c=d=0$ define the Sasaki metric $\widetilde{G}_{S}$ ). In [Abbassi et al. 2008] the harmonicity of the map $M \rightarrow T_{1} M$ defined by a unit vector field was studied in the case when the unit tangent sphere bundle $T_{1} M$ is equipped with an arbitrary Riemannian $g$-natural metric $\widetilde{G}$.

Han and Yim [1998] showed their Theorem A by using the property that the Hopf fibration $S^{3} \rightarrow \mathbb{C P}^{1}$ is the unique fibration of the round three-sphere by great circles such that the fibres are parallel (in the sense of having constance distance from another) [Escobales 1975]. In this paper, by using a different method, we extend Theorem A by replacing the unit sphere $S^{3}$ by a Riemannian three-manifold of constant sectional curvature $k \neq 0$ and the Sasaki metric $\widetilde{G}_{s}$ by an arbitrary Riemannian $g$-natural metric $\widetilde{G}_{a, d, c}$ that is a deformation depending on three real parameters of the Sasaki metric $\widetilde{\widetilde{G}}_{s}$; such a deformation preserves the property (of the Sasaki metric) that horizontal and vertical lifts are orthogonal. We do not assume that $M$ is compact. So, in particular, $M$ may be an open (connected) subset of the sphere $S^{3}$. More precisely we get this:

Theorem 1.1. Let $(M, g)$ be a Riemannian three-manifold of constant sectional curvature $\kappa \neq 0$ and $T_{1} M$ its unit tangent sphere bundle equipped with a Riemannian g-natural metric $\widetilde{G}_{a, d, c}$ with $d \neq-\kappa a($ and $b=0)$. Let $\xi$ be in $\mathfrak{X}^{1}(M)$. Then $\xi:(M, g) \rightarrow\left(T_{1} M, \widetilde{G}_{a, d, c}\right)$ is a harmonic map if and only if $\xi$ is Killing and $\kappa>0$.

Theorem 1.1 has an immediate consequence:

Corollary 1.2. Han and Yim's theorem is invariant under a three-parameter deformation of the Sasaki metric on $T_{1} M$. 
In the case of the hyperbolic space $H^{n}(-k)$ for $k>0$, it is an open question whether some unit vector field exists (of course, non-Killing) that defines a harmonic map from $H^{n}(-k)$ to $\left(T_{1} H^{n}(-k), \widetilde{G}_{s}\right)$. From Theorem 1.1 we have the following nonexistence result in dimension three.

Corollary 1.3. Let $H^{3}(-k)$ be the hyperbolic three-space. Then there does not exist a unit vector field that defines a harmonic map between the Riemannian manifolds $H^{3}(-k)$ and $\left(T_{1} H^{3}(-k), \widetilde{G}_{s}\right)$. Such a result is invariant under a threeparameter deformation of the Sasaki metric on $T_{1} H^{3}(-k)$.

On a flat three-space and on the sphere $S^{3}$ equipped with a metric of nonconstant sectional curvature we give examples of unit vector fields that are not Killing but define harmonic maps; see Example 3.1.

In their paper Han and Yim [Han and Yim 1998, page 84] posed the question of whether Theorem A is true for higher dimensions spheres. Here, we consider the following question (which generalizes the Han and Yim's question): Let $(M, g)$ be a real space form of constant sectional curvature $\kappa>0$. Are the unit Killing vector fields on $M$ the only unit vector fields that define harmonic maps from $(M, g)$ to $\left(T_{1} M, \widetilde{G}\right)$ ? If $M$ is not homeomorphic to the sphere, we get a positive answer:

Theorem 1.4. Let $(M, g)$ be a real space form of constant positive sectional curvature $\kappa$, with $\operatorname{dim} M=2 m+1$. Suppose that $M$ is not homeomorphic to the sphere $S^{2 m+1}$. Let $\left(T^{1} M, \widetilde{G}\right)$ be its unit tangent sphere bundle equipped with a Riemannian $g$-natural metric $\widetilde{G}=\widetilde{G}_{a, b, c, d}$ with $b \neq 0$ and $d \neq-k a$. Let $\xi \in \mathfrak{X}^{1}(M)$. Then

(i) $\xi:(M, g) \rightarrow\left(T^{1} M, \widetilde{G}\right)$ is a harmonic map if and only if $\xi$ is Killing;

(ii) if $\xi$ is a solenoidal (that is, a divergence free) unit vector field, then

$$
\xi:(M, g) \rightarrow\left(T^{1} M, \widetilde{G}\right)
$$

is a harmonic map if and only if $\xi$ has minimum energy $E_{\widetilde{G}}: \mathfrak{X}^{1}(M) \rightarrow \mathbb{R}$.

Remark 1.5. Brito and Salvai [2004, Proposition 1] proved that if $M$ is a compact Riemannian manifold and $\xi$ is a unit Killing vector field eigenvector of the Ricci operator and is of minimum Ricci curvature, then $\xi$ has minimum energy among all solenoidal unit vector fields.

In the final Section 5, we remark that the main results of [Brito 2000; Perrone 2008], related to the energy restricted to $\mathfrak{X}^{1}(M)$, are invariant under a fourparameter deformation of the Sasaki metric on $T_{1} M$.

\section{Preliminaries}

Let $(M, g)$ be an $n$-dimensional Riemannian manifold and $\nabla$ its Levi-Civita connection. We denote by $R$ the Riemannian curvature tensor of $(M, g)$ with the sign 
convention

$$
R(X, Y) Z=-\nabla_{X} \nabla_{Y} Z+\nabla_{Y} \nabla_{X} Z+\nabla_{[X, Y]} Z \text { for all } X, Y, Z \in \mathfrak{X}(M) .
$$

We denote by Ric the Ricci tensor of type $(0,2)$, by $Q$ the corresponding endomorphism field, and by $r$ the scalar curvature.

At any point $(x, u)$ of the tangent bundle $T M$, the tangent space of $T M$ splits into horizontal and vertical subspaces with respect to $\nabla$ :

$$
(T M)_{(x, u)}=\mathscr{H}_{(x, u)} \oplus \mathscr{V}_{(x, u)} .
$$

The Riemannian g-natural metrics form a large family of Riemannian metrics on $T M$. These metrics depend on several smooth functions from $\mathbb{R}^{+}=[0,+\infty)$ to $\mathbb{R}$, and, as their name suggests, they arise from a natural construction starting from a Riemannian metric $g$ over $M$; see [Abbassi and Sarih 2005; Kolár et al. 1993]. Given an arbitrary $g$-natural metric $G$ on the tangent bundle $T M$ of a Riemannian manifold $(M, g)$, there are six smooth functions $\alpha_{i}, \beta_{i}: \mathbb{R}^{+} \rightarrow \mathbb{R}$ for $i=1,2,3$ such that for every $u, X, Y \in M_{x}$, we have

$$
\left\{\begin{aligned}
G_{(x, u)}\left(X^{h}, Y^{h}\right) & =\left(\alpha_{1}+\alpha_{3}\right)\left(r^{2}\right) g_{x}(X, Y) \\
& +\left(\beta_{1}+\beta_{3}\right)\left(r^{2}\right) g_{x}(X, u) g_{x}(Y, u), \\
G_{(x, u)}\left(X^{h}, Y^{v}\right) & =G_{(x, u)}\left(X^{v}, Y^{h}\right) \\
& =\alpha_{2}\left(r^{2}\right) g_{x}(X, Y)+\beta_{2}\left(r^{2}\right) g_{x}(X, u) g_{x}(Y, u), \\
G_{(x, u)}\left(X^{v}, Y^{v}\right) & =\alpha_{1}\left(r^{2}\right) g_{x}(X, Y)+\beta_{1}\left(r^{2}\right) g_{x}(X, u) g_{x}(Y, u),
\end{aligned}\right.
$$

where $r^{2}=g_{x}(u, u)$. Put

$$
\begin{aligned}
& \phi_{i}(t)=\alpha_{i}(t)+t \beta_{i}(t), \quad \alpha(t)=\alpha_{1}(t)\left(\alpha_{1}+\alpha_{3}\right)(t)-\alpha_{2}^{2}(t), \\
& \phi(t)=\phi_{1}(t)\left(\phi_{1}+\phi_{3}\right)(t)-\phi_{2}^{2}(t)
\end{aligned}
$$

for all $t \in \mathbb{R}^{+}$. Then, a $g$-natural metric $G$ on $T M$ is Riemannian if and only if the inequalities

$$
\begin{aligned}
\alpha_{1}(t) & >0, & \phi_{1}(t) & >0, \\
\alpha(t) & >0, & \phi(t) & >0
\end{aligned}
$$

hold for all $t \in \mathbb{R}^{+}$. Notice that the Sasaki metric $G_{s}$, the Cheeger-Gromoll metric and other Riemannian metrics on $T M$ belong to the class of Riemannian $g$-natural metrics on $T M$ for which horizontal and vertical distributions are mutually orthogonal (that is, $\alpha_{2}=\beta_{2}=0$ ).

Next, the unit tangent sphere bundle over a Riemannian manifold $(M, g)$ is the hypersurface $T_{1} M=\left\{(x, u) \in T M \mid g_{x}(u, u)=1\right\}$. The tangent space of $T_{1} M$ at a 
point $(x, u) \in T_{1} M$ is given by

$$
\left(T_{1} M\right)_{(x, u)}=\left\{X^{h}+Y^{v}: X \in M_{x}, Y \in\{u\}^{\perp} \subset M_{x}\right\} .
$$

We call $g$-natural metrics on $T_{1} M$ the restrictions of $g$-natural metrics of $T M$ to its hypersurface $T_{1} M$. These metrics possess a simpler form. Precisely, taking in account of (2-3), a Riemannian $g$-natural metric $\widetilde{G}$ on $T_{1} M$ is induced by a Riemannian $g$-natural $G$ on $T M$ of the form

$$
\left\{\begin{aligned}
G_{(x, u)}\left(X_{1}^{h}, X_{2}^{h}\right)= & \left(\alpha_{1}+\alpha_{3}\right)\left(r^{2}\right) g_{x}\left(X_{1}, X_{2}\right) \\
& +\left(\beta_{1}+\beta_{3}\right)\left(r^{2}\right) g_{x}\left(X_{1}, u\right) g_{x}\left(X_{2}, u\right), \\
G_{(x, u)}\left(X_{1}^{h}, Y_{1}^{v}\right)= & \alpha_{2}\left(r^{2}\right) g_{x}\left(X_{1}, Y_{1}\right), \\
G_{(x, u)}\left(Y_{1}^{v}, Y_{2}^{v}\right)= & \alpha_{1}\left(r^{2}\right) g_{x}\left(Y_{1}, Y_{2}\right),
\end{aligned}\right.
$$

for all $X_{1}, X_{2} \in M_{x}$ and $Y_{1}, Y_{2} \in\{u\}^{\perp}$; see [Abbassi and Sarih 2005; Abbassi and Calvaruso 2007] and references therein. In other words, $\widetilde{G}$ on $T_{1} M$ is necessarily induced by a Riemannian $g$-natural $G$ on $T M$ of the form (2-1) with

$$
\alpha_{1}=a, \quad \alpha_{2}=b, \quad \alpha_{3}=c, \quad \beta_{1}=\beta_{2}=0, \quad \beta_{3}=\beta,
$$

where $a, b, c$ are three real constants and $\beta:[0, \infty) \rightarrow \mathbb{R}$ is a smooth function. Such a metric $\widetilde{G}$ on $T_{1} M$ only depends on the value $d:=\beta(1)$ of $\beta$ at 1 . From (2-2) and (2-5), it follows that $\widetilde{G}$ is Riemannian if and only if the constants $a, b, c$ and $d$ satisfy the inequalities

$$
a>0, \quad \alpha:=a(a+c)-b^{2}>0, \quad \phi:=a(a+c+d)-b^{2}>0 .
$$

We denote by $\widetilde{G}_{a, b, c, d}$ such a Riemannian $g$-natural metric on $T_{1} M$ and define $\widetilde{G}_{a, c, d}=\widetilde{G}_{a, 0, c, d}$. We can consider $\widetilde{G}_{a, b, c, d}$ to be a deformation on four parameters of the Sasaki metric $\widetilde{G}_{s}=\widetilde{G}_{1,0,0,0}$. It should be noted that, by (2-4), horizontal and vertical lifts are orthogonal with respect to $\widetilde{G}$ if and only if $b=0$.

Now let $(M, g)$ be a compact Riemannian manifold of dimension $n$. A unit vector field $V$ defines a mapping from $(M, g)$ to $T_{1} M$ equipped with a Riemannian $g$-natural metric $\widetilde{G}$. The energy functional $E_{\widetilde{G}}: \mathfrak{X}^{1}(M) \rightarrow \mathbb{R}$ is defined by $E_{\widetilde{G}}(V)=\int_{M} e(V) d v_{g}$, where $e(V)$ is the energy density of $V$ and is given by [Abbassi et al. 2008]

$$
2 e(V)=n(a+c)+d+a\|\nabla V\|^{2}+2 b \operatorname{div} V .
$$

So, integrating over $M$ we get

$$
E_{\widetilde{G}}(V)=\frac{1}{2}(n(a+c)+d) \operatorname{vol}(M, g)+\frac{a}{2} \int_{M}\|\nabla V\|^{2} d v_{g}
$$


In [Abbassi et al. 2008], it was proved that $V:(M, g) \rightarrow\left(T_{1} M, \widetilde{G}\right)$ is a harmonic map if and only if

$$
\begin{aligned}
& \bar{\Delta} V \text { is collinear to } V \text {, and } \\
& b Q V+a \operatorname{tr}(R(\nabla . V, V) \cdot)=\left(b\|\nabla V\|^{2}-d \operatorname{div} V\right) V+d \nabla_{V} V,
\end{aligned}
$$

where $\bar{\Delta} V=-\operatorname{tr} \nabla^{2} V$ is the rough Laplacian at $V$. Such conditions have a tensorial character; hence they may also be considered to define a harmonic map on noncompact manifolds. In the special case of the Sasaki metric $\widetilde{G}_{s}$, that is, $a=1$ and $b=c=d=0,(2-8)$ gives a result of [Han and Yim 1998]. If $V \in \mathfrak{X}^{1}(M)$ is a unit Killing vector field, then [Poor 1981, page 169]

$$
\nabla_{V} V=0, \quad \operatorname{div} V=0, \quad \bar{\Delta} V=Q V,
$$

and, $V$ being a unit vector field, $g(Q V, V)=g(\bar{\Delta} V, V)=\|\nabla V\|^{2}$. Then, since $a>0$, (2-8) gives this:

Proposition 2-9. If $V \in \mathfrak{X}^{1}(M)$ is a unit Killing vector field, then the harmonicity of the map $V:(M, g) \rightarrow\left(T_{1} M, \tilde{G}_{s}\right)$ implies that of $V:(M, g) \rightarrow\left(T_{1} M, \widetilde{G}\right)$ for any $\widetilde{G}$.

\section{Proof of Theorem 1.1}

Let $(M, g)$ be a Riemannian three-manifold of constant sectional curvature $\kappa \neq 0$ and $T_{1} M$ its unit tangent sphere bundle equipped with a Riemannian $g$-natural metric $\widetilde{G}$.

Assume that $\xi$ is a unit Killing vector field. Since $M$ has constant sectional curvature, $\xi:(M, g) \rightarrow\left(T_{1} M, \tilde{G}_{s}\right)$ is a harmonic map, and hence, by Proposition 2-9, we obtain that $\xi:(M, g) \rightarrow\left(T_{1} M, \widetilde{G}\right)$ is a harmonic map for any $\widetilde{G}$.

Conversely, we suppose that $\xi:(M, g) \rightarrow\left(T^{1} M, \widetilde{G}_{a, d, c}\right)$ is a harmonic map, where $\widetilde{G}_{a, d, c}$ is a Riemannian $g$-natural metric with $d \neq-\kappa a$ (and $b=0$ ). By (2-8), we obtain

$$
\begin{aligned}
\bar{\Delta} \xi & =\|\xi\|^{2} \xi \\
\operatorname{tr}(R(\nabla . \xi, \xi) \cdot) & =-\frac{d}{a}\left((\operatorname{div} \xi) \xi-\nabla_{\xi} \xi\right) .
\end{aligned}
$$

Now $\operatorname{tr}(R(\nabla . \xi, \xi) \cdot)=k\left((\operatorname{div} \xi) \xi-\nabla_{\xi} \xi\right)$ since $M$ has constant sectional curvature $k$. Then $k \neq-d / a$ and condition (3-1a) imply

$$
\operatorname{div} \xi=0 \quad \text { and } \quad \nabla_{\xi} \xi=0 .
$$

Put $\tau:=\mathscr{L}_{\xi} g$, where $\mathscr{L}_{\xi}$ denotes the Lie derivative, that is,

$$
\tau(X, Y)=g\left(\nabla_{X} \xi, Y\right)+g\left(X, \nabla_{Y} \xi\right) .
$$


Since $\tau$ is a symmetric tensor of type $(0,2)$, we can define the corresponding symmetric $(1,1)$ tensor $h$ through $\tau(X, Y)=g(h X, Y)$. Then, from (3-2),

$$
g(h \xi, Y)=\tau(\xi, Y)=g\left(\nabla_{\xi} \xi, Y\right)+g\left(\xi, \nabla_{Y} \xi\right)=\frac{1}{2} Y\left(\|\xi\|^{2}\right)=0
$$

and hence $h \xi=0$. So, we can consider a local orthonormal basis $\left\{e_{1}, e_{2}, e_{3}=\xi\right\}$ of eigenvectors of $h$, with $h \xi=0, h e_{1}=\lambda_{1} e_{1}$ and $h e_{2}=\lambda_{2} e_{2}$. Since

$$
\begin{aligned}
\operatorname{div} \xi & =g\left(\nabla_{\xi} \xi, \xi\right)+g\left(\nabla_{e_{1}} \xi, e_{1}\right)+g\left(\nabla_{e_{2}} \xi, e_{2}\right) \\
& =\frac{1}{2} g\left(h e_{1}, e_{1}\right)+\frac{1}{2} g\left(h e_{2}, e_{2}\right)=\frac{1}{2}\left(\lambda_{1}+\lambda_{2}\right),
\end{aligned}
$$

from (3-2) we get $\lambda_{2}=-\lambda_{1}$. Therefore $\xi$ is Killing if and only if $\lambda_{1}=0$. Put

$$
\begin{aligned}
& f_{1}=\frac{1}{2} \lambda_{1}, \quad f_{2}=g\left(\nabla_{e_{1}} \xi, e_{2}\right), \quad f_{3}=g\left(\nabla_{e_{1}} e_{2}, e_{1}\right), \\
& f_{4}=g\left(\nabla_{e_{2}} e_{2}, e_{1}\right), \quad f_{5}=g\left(\nabla_{\xi} e_{1}, e_{2}\right) \text {. }
\end{aligned}
$$

Then, we have the following list of covariant derivatives:

$$
\begin{aligned}
\nabla_{e_{1}} \xi & =f_{1} e_{1}+f_{2} e_{2} \\
\nabla_{\xi} \xi & =0 \\
\nabla_{e_{2}} \xi & =g\left(\nabla_{e_{2}} \xi, e_{1}\right) e_{1}+g\left(\nabla_{e_{2}} \xi, e_{2}\right) e_{2} \\
& =\left(g\left(h e_{1}, e_{2}\right)-g\left(\nabla_{e_{1}} \xi, e_{2}\right)\right) e_{1}+\frac{1}{2} g\left(h e_{2}, e_{2}\right) e_{2}=-f_{2} e_{1}-f_{1} e_{2}, \\
\nabla_{e_{1}} e_{1} & =g\left(\nabla_{e_{1}} e_{1}, \xi\right) \xi+g\left(\nabla_{e_{1}} e_{1}, e_{2}\right) e_{2} \\
& =-g\left(\nabla_{e_{1}} \xi, e_{1}\right) \xi-g\left(\nabla_{e_{1}} e_{2}, e_{1}\right) e_{2}=-f_{1} \xi-f_{3} e_{2}, \\
\nabla_{e_{2}} e_{2} & =g\left(\nabla_{e_{2}} e_{2}, \xi\right) \xi+g\left(\nabla_{e_{2}} e_{2}, e_{1}\right) e_{1} \\
& =-g\left(\nabla_{e_{2}} \xi, e_{2}\right) \xi+f_{4} e_{1}=-\frac{1}{2} g\left(h e_{2} e_{2}\right) \xi+f_{4} e_{1}=f_{1} \xi+f_{4} e_{1}, \\
\nabla_{e_{1}} e_{2} & =g\left(\nabla_{e_{1}} e_{2}, \xi\right) \xi+g\left(\nabla_{e_{1}} e_{2}, e_{1}\right) e_{1} \\
& =-g\left(\nabla_{e_{1}} \xi, e_{2}\right) \xi+f_{3} e_{1}=-f_{2} \xi+f_{3} e_{1}, \\
\nabla_{e_{2}} e_{1} & =g\left(\nabla_{e_{2}} e_{1}, \xi\right) \xi+g\left(\nabla_{e_{2}} e_{1}, e_{2}\right) e_{2}=-g\left(\nabla_{e_{2}} \xi, e_{1}\right) \xi-g\left(\nabla_{e_{2}} e_{2}, e_{1}\right) e_{2} \\
& =-\left(g\left(h e_{1}, e_{2}\right)-g\left(\nabla_{e_{1}} \xi, e_{2}\right)\right) \xi-f_{4} e_{2}=f_{2} \xi-f_{4} e_{2}, \\
\nabla_{\xi} e_{1} & =g\left(\nabla_{\xi} e_{1}, \xi\right) \xi+g\left(\nabla_{\xi} e_{1}, e_{2}\right) e_{2}=-g\left(\nabla_{\xi} \xi, e_{1}\right) \xi+f_{5} e_{2}=f_{5} e_{2}, \\
\nabla_{\xi} e_{2} & =g\left(\nabla_{\xi} e_{2}, \xi\right) \xi+g\left(\nabla_{\xi} e_{2}, e_{1}\right) e_{1}=-g\left(\nabla_{\xi} \xi, e_{2}\right) \xi-f_{5} e_{1}=-f_{5} e_{1} .
\end{aligned}
$$

Moreover,

$$
\begin{aligned}
& {\left[e_{1}, \xi\right]=f_{1} e_{1}+\left(f_{2}-f_{5}\right) e_{2},} \\
& {\left[e_{2}, \xi\right]=\left(f_{5}-f_{2}\right) e_{1}-f_{1} e_{2}, \quad\left[e_{1}, e_{2}\right]=-2 f_{2} \xi+f_{3} e_{1}+f_{4} e_{2} .}
\end{aligned}
$$


By using the list of covariant derivatives, we get

$$
\left\{\begin{aligned}
R\left(e_{1}, \xi\right) \xi= & -\nabla_{e_{1}} \nabla_{\xi} \xi+\nabla_{\xi} \nabla_{e_{1}} \xi+\nabla_{\left[e_{1}, \xi\right]} \xi \\
= & \left(f_{1}^{2}-f_{2}^{2}+\xi\left(f_{1}\right) g\right) e_{1}+\left(2 f_{1} f_{5}+\xi\left(f_{2}\right)\right) e_{2}, \\
R\left(e_{2}, \xi\right) \xi= & -\nabla_{e_{2}} \nabla_{\xi} \xi+\nabla_{\xi} \nabla_{e_{2}} \xi+\nabla_{\left[e_{2}, \xi\right]} \xi \\
= & \left(2 f_{1} f_{5}-\xi\left(f_{2}\right)\right) e_{1}+\left(f_{1}^{2}-f_{2}^{2}-\xi\left(f_{1}\right)\right) e_{2}, \\
R\left(e_{1}, e_{2}\right) \xi= & -\nabla_{e_{1}} \nabla_{e_{2}} \xi+\nabla_{e_{2}} \nabla_{e_{1}} \xi+\nabla_{\left[e_{1}, e_{2}\right]} \xi \\
= & \left(e_{1}\left(f_{2}\right)+e_{2}\left(f_{1}\right)+2 f_{1} f_{3}\right) e_{1} \\
& +\left(e_{1}\left(f_{1}\right)+e_{2}\left(f_{2}\right)-2 f_{1} f_{4}\right) e_{2}, \\
R\left(e_{1}, e_{2}\right) e_{1}= & -\nabla_{e_{1}} \nabla_{e_{2}} e_{1}+\nabla_{e_{2}} \nabla_{e_{1}} e_{1}+\nabla_{\left[e_{1}, e_{2}\right]} e_{1} \\
= & -\left(e_{1}\left(f_{2}\right)+e_{2}\left(f_{1}\right)+2 f_{1} f_{3}\right) \xi \\
& +\left(f_{1}^{2}-f_{2}^{2}+e_{1}\left(f_{4}\right)-e_{2}\left(f_{3}\right)-2 f_{2} f_{5}-f_{3}^{2}-f_{4}^{2}\right) e_{2} .
\end{aligned}\right.
$$

Besides, $R(X, Y) Z=\kappa(g(X, Z) Y-g(Y, Z) X)$ gives

$$
\begin{aligned}
R\left(e_{1}, \xi, e_{1}, \xi\right) & =R\left(e_{2}, \xi, e_{2}, \xi\right)=R\left(e_{1}, e_{2}, e_{1}, e_{2}\right)=\kappa, \\
R\left(e_{1}, \xi\right) e_{2} & =R\left(e_{1}, e_{2}\right) \xi=R\left(e_{2}, \xi\right) e_{1}=0 .
\end{aligned}
$$

From (3-3) and (3-4), we obtain

$$
\begin{array}{rlrl}
f_{2}^{2}-f_{1}^{2}-\xi\left(f_{1}\right) & =\kappa, & 2 f_{1} f_{5}+\xi\left(f_{2}\right) & =0, \\
f_{2}^{2}-f_{1}^{2}+\xi\left(f_{1}\right) & =\kappa, & 2 f_{1} f_{5}-\xi\left(f_{2}\right) & =0, \\
e_{1}\left(f_{2}\right)+e_{2}\left(f_{1}\right)+2 f_{1} f_{3} & =0, & e_{1}\left(f_{1}\right)+e_{2}\left(f_{2}\right)-2 f_{1} f_{4} & =0, \\
f_{1}^{2}-f_{2}^{2}+e_{1}\left(f_{4}\right)-e_{2}\left(f_{3}\right)-2 f_{2} f_{5}-f_{3}^{2}-f_{4}^{2} & =\kappa .
\end{array}
$$

From (3-5) and (3-6), we get

$$
f_{2}^{2}-f_{1}^{2}=\kappa, \quad f_{1} f_{5}=0, \quad \xi\left(f_{1}\right)=\xi\left(f_{2}\right)=0 .
$$

Using (3-9), (3-8) becomes

$$
e_{1}\left(f_{4}\right)-e_{2}\left(f_{3}\right)-2 f_{2} f_{5}-f_{3}^{2}-f_{4}^{2}=2 k .
$$

Now we compute $\bar{\Delta} \xi$. Since

$$
\begin{aligned}
\nabla_{e_{1}} \nabla_{e_{1}} \xi & =\nabla_{e_{1}}\left(f_{1} e_{1}+f_{2} e_{2}\right)=e_{1}\left(f_{1}\right) e_{1}+f_{1} \nabla_{e_{1}} e_{1}+e_{1}\left(f_{2}\right) e_{2}+f_{2} \nabla_{e_{1}} e_{2} \\
& =-\left(f_{1}^{2}+f_{2}^{2}\right) \xi+\left(e_{1}\left(f_{1}\right)+f_{2} f_{3}\right) e_{1}+\left(e_{1}\left(f_{2}\right)-f_{1} f_{3}\right) e_{2}, \\
\nabla_{e_{2}} \nabla_{e_{2}} \xi & =\nabla_{e_{2}}\left(-f_{2} e_{1}-f_{1} e_{2}\right)=-e_{2}\left(f_{2}\right) e_{1}-f_{2} \nabla_{e_{2}} e_{1}-e_{2}\left(f_{1}\right) e_{2}-f_{1} \nabla_{e_{2}} e_{2} \\
& =-\left(f_{1}^{2}+f_{2}^{2}\right) \xi-\left(e_{2}\left(f_{2}\right)+f_{1} f_{4}\right) e_{1}-\left(e_{2}\left(f_{1}\right)-f_{2} f_{4}\right) e_{2},
\end{aligned}
$$


and

$$
\begin{aligned}
& -\nabla_{\nabla_{e_{1}} e_{1}} \xi=f_{3} \nabla_{e_{2}} \xi=-f_{3}\left(f_{2} e_{1}+f_{1} e_{2}\right), \\
& -\nabla_{\nabla_{e_{2}} e_{2}} \xi=-f_{4} \nabla_{e_{1}} \xi=-f_{4}\left(f_{1} e_{1}+f_{2} e_{2}\right),
\end{aligned}
$$

we have

$$
\begin{aligned}
-\bar{\Delta} \xi= & \operatorname{tr} \nabla^{2} \xi \\
= & \nabla_{e_{1}} \nabla_{e_{1}} \xi+\nabla_{e_{2}} \nabla_{e_{2}} \xi+\nabla_{\xi} \nabla_{\xi} \xi-\nabla_{\nabla_{e_{1}} e_{1}} \xi-\nabla_{\nabla_{e_{2}} e_{2}} \xi-\nabla_{\nabla_{\xi} \xi} \xi \\
= & -\|\nabla \xi\|^{2} \xi+\left(e_{1}\left(f_{1}\right)-e_{2}\left(f_{2}\right)-2 f_{1} f_{4}\right) e_{1} \\
& \quad+\left(e_{1}\left(f_{2}\right)-e_{2}\left(f_{1}\right)-2 f_{1} f_{3}\right) e_{2},
\end{aligned}
$$

where $\|\nabla \xi\|^{2}=2\left(f_{1}^{2}+f_{2}^{2}\right)$. Because of (3-1a), from (3-11) we get

$$
e_{1}\left(f_{1}\right)-e_{2}\left(f_{2}\right)=2 f_{1} f_{4} \text { and } e_{1}\left(f_{2}\right)-e_{2}\left(f_{1}\right)=2 f_{1} f_{3} .
$$

Combining (3-7) and (3-12), we have

$$
e_{1}\left(f_{2}\right)=e_{2}\left(f_{2}\right)=0 .
$$

Moreover, (3-9) implies $\xi\left(f_{2}\right)=0$ and $f_{1}^{2}=f_{2}^{2}-\kappa$. So $f_{1}$ and $f_{2}$ are constant. If $f_{1}=$ const $\neq 0$, from (3-5) and (3-7) we have $f_{3}=f_{4}=f_{5}=0$ which, by (3-8), imply $\kappa=0$. Since $\kappa \neq 0$, we conclude that $f_{1}=0$ and hence $\xi$ is Killing and, by (3-6), we have $\kappa=f_{2}^{2}>0$.

Example 3.1 (Non-Killing unit vector fields that define harmonic maps). Let $\mathfrak{g}$ be a three-dimensional Lie algebra. Introduce a basis $\left(e_{1}, e_{2}, e_{3}\right)$ for $\mathfrak{g}$, and for real numbers $\lambda_{1}, \lambda_{2}$ and $\lambda_{3}$, define the Lie bracket by

$$
\left[e_{2}, e_{3}\right]=\lambda_{1} e_{1}, \quad\left[e_{3}, e_{1}\right]=\lambda_{2} e_{2}, \quad\left[e_{1}, e_{2}\right]=\lambda_{3} e_{3} .
$$

On the associated Lie group $\mathscr{G}$, define a metric $g$ by left translation of the basis $\left(e_{1}, e_{2}, e_{3}\right)$, taken as orthogonal at the identity. Suppose $\mathscr{G}$ to be simply connected; otherwise we consider its universal covering. Let $\left(\vartheta^{1}, \vartheta^{2}, \vartheta^{3}\right)$ be the metric dual one-forms of $\left(e_{1}, e_{2}, e_{3}\right)$. If $\lambda_{i} \neq 0$, then $\vartheta^{i}$ is a contact form, that is, $\vartheta^{i} \wedge \mathrm{d} \vartheta^{i} \neq 0$, and $e_{i}$ is the corresponding Reeb vector field. Assuming $\lambda_{1}=2$ and defining $\varphi$ by $\varphi\left(e_{1}\right)=0, \varphi\left(e_{2}\right)=e_{3}$ and $\varphi\left(e_{3}\right)=-e_{2}$, we have $d \vartheta^{1}=g(\cdot, \varphi \cdot)$. Then $\left(\eta=\vartheta^{1}, g, \varphi, \xi=e_{1}\right)$ is a contact metric structure on $G_{\text {; }}$ see [Blair 2002]. Such a structure is a $(\kappa, \mu)$-structure, and it is Sasakian, that is, $\xi$ is Killing if and only if $\lambda_{2}=\lambda_{3}$; see [Perrone 1998], which also gives that

(a) if $\lambda_{2}>0, \lambda_{3}>0$ and $\lambda_{2} \neq \lambda_{3}$, then the group $\mathscr{G}$ is the three-sphere group $S U(2), \xi$ is not Killing, and the metric has nonconstant sectional curvature;

(b) if $\lambda_{2}=0$ and $\lambda_{3}>0$, then $\varphi$ is $\widetilde{E}_{2}$ the universal covering of the group of rigid motions of Euclidean 2-space, $\xi$ is not Killing, and the Ricci tensor is given by 
$\operatorname{Ric}_{i j}=0$ for $i \neq j, \operatorname{Ric}_{11}=-\operatorname{Ric}_{33}=\left(4-\lambda_{3}^{2}\right) / 2$ and $\operatorname{Ric}_{22}=-\left(\lambda_{3}-2\right)^{2} / 2$; hence the scalar curvature $r \leq 0$, and the metric is flat if and only if $\lambda_{3}=2$.

In both cases from [Perrone 2003, Theorem 1.1], we have that $\xi$ defines a harmonic map between the Riemannian manifolds $(\mathscr{G}, g)$ and $\left(T_{1} \mathscr{G}, \widetilde{G}_{s}\right)$, that is, $Q \xi$ is collinear to $\xi$ and $\operatorname{tr}(R(\nabla . \xi, \xi) \cdot)=0$. On the other hand, in [Abbassi et al. 2008, Theorem 7] we proved this:

Theorem 3.2. Let $\left(M^{2 m+1}, \eta, g\right)$ be a contact metric manifold and $\widetilde{G}$ an arbitrary Riemannian g-natural metric on $T_{1} M$. Then $\xi:(M, g) \rightarrow\left(T_{1} M, \widetilde{G}\right)$ is a harmonic map if and only if

$$
a \operatorname{tr}(R(\nabla \cdot \xi, \xi) \cdot)=-2 b\left(\|\nabla \xi\|^{2}-2 m\right) \xi \quad \text { and } \quad Q \xi \text { is collinear to } \xi .
$$

Since $\|\nabla \xi\|^{2}-2 m=0$ if and only if $\xi$ is Killing [Blair 2002, Lemma 6.2, page 67], from Theorem 3.2 we deduce that $\xi:(\mathscr{G}, g) \rightarrow\left(T_{1} \mathscr{G}, \widetilde{G}\right)$ defines a harmonic map if and only if $b=0$. Thus $\xi:(\mathscr{G}, g) \rightarrow\left(T_{1} \mathscr{G}, \widetilde{G}_{a, d, c}\right)$ is a harmonic map.

\section{Proof of Theorem 1.4}

Let $(M, g)$ be a real space form of positive constant sectional curvature $\kappa>0$, with $\pi_{1}(M) \neq 0$. Then $(M, g)$ is isometric to the spherical space form $\left(S^{2 m+1} / \Gamma, g\right)$, where $\Gamma \neq\{I d\}$ is a finite subgroup of $O(2 m+2)$ in which only the identity element has +1 as an eigenvalue, and $g$ is the Riemannian metric on the quotient space $S^{2 m+1} / \Gamma$ induced by the canonical metric.

We first prove Theorem 1.4(i). If $\xi$ is a unit Killing vector field on $M$, then as in the 3-dimensional case, $\xi:(M, g) \rightarrow\left(T_{1} M, \tilde{G}_{S}\right)$ is a harmonic map and, by Proposition 2-9, $\xi:(M, g) \rightarrow\left(T_{1} M, \widetilde{G}\right)$ is a harmonic map for any $\widetilde{G}$.

Vice versa, let $V$ be in $\mathfrak{X}^{1}(M)$. Suppose that $V:(M, g) \rightarrow\left(T^{1} M, \widetilde{G}\right)$ is a harmonic map, where $\widetilde{G}$ is a Riemannian $g$-natural metric on $T^{1} M$ with $b \neq 0$ and $d+a k \neq 0$. Then, by (2-8), $\bar{\Delta} V=\|\nabla V\|^{2} V$ and

$$
b Q V=-a(\operatorname{tr} R(\nabla . V, V) \cdot)+b\|\nabla V\|^{2} V+d\left(\nabla_{V} V-(\operatorname{div} V) V\right) .
$$

Since $M$ has constant sectional curvature $\kappa,(4-1)$ becomes

$$
b\left(2 m \kappa-\|\nabla V\|^{2}\right) V=(d+a \kappa)\left(\nabla_{V} V-(\operatorname{div} V) V\right) .
$$

This formula implies $b\left(\|\nabla V\|^{2}-2 m \kappa\right)=(d+a \kappa) \operatorname{div} V$, and hence

$$
b \int_{M}\left(\|\nabla V\|^{2}-2 m \kappa\right) d v_{g}=0 .
$$


Recall that, if $f:(M, g) \rightarrow(N, h)$ is a harmonic map, the Hessian form of the energy $E$ at $f$ is defined by the second variation formula [Smith 1975]:

$$
(\operatorname{Hess} E)_{f}(X, X)=\left.\frac{\mathrm{d}^{2}}{\mathrm{~d} t^{2}} E\left(f_{t}\right)\right|_{t=0}=\int_{M} h\left(X, J_{f} X\right) d v_{g},
$$

where $X$ is a vector field along $f$. The operator $J_{f}$, called the Jacobi operator of $f$, is a second order self adjoint elliptic differential operator acting on the space $\Gamma\left(f^{-1} T N\right)$ of the vector fields along $f$, and is defined by

$$
J_{f} X=\bar{\Delta}_{f} X-\operatorname{Ric}_{f} X .
$$

The operator $\bar{\Delta}_{f}$, called the rough Laplacian along $f$, is defined by

$$
\bar{\Delta}_{f} X=-\sum_{i=1}^{n}\left(\bar{\nabla}_{e_{i}} \bar{\nabla}_{e_{i}} X-\bar{\nabla}_{\nabla_{e_{i}} e_{i}} X\right) \quad \text { for } X \in \Gamma\left(f^{-1} T N\right),
$$

where $\bar{\nabla}$ is the connection (on the vector bundle $f^{-1} T N$ ) induced by the LeviCivita connection of $(N, h)$, and $\left\{e_{i}\right\}_{i=1, \ldots, n}$ is a local orthonormal frame on $M$. Moreover, denoting by $R_{h}$ the curvature tensor of $(N, h)$,

$$
\operatorname{Ric}_{f} X=\sum_{i=1}^{n} R_{h}\left(f_{*} e_{i}, X\right) f_{*} e_{i} .
$$

A harmonic map $f$ is said to be stable if (Hess $E)_{f}$ is semidefinite positive or, equivalently, if the eigenvalues of the Jacobi operator are nonnegative. The identity map Id $:(M, g) \rightarrow(M, g)$ is a trivial example of a harmonic map. From (4-4)(4-6) we readily deduce that the second variation formula of the energy for $I d$ is given by

$$
(\operatorname{Hess} E)_{I d}(X, X)=\int_{M} g\left(J_{I d} X, X\right) d v_{g}=\int_{M} g(\bar{\Delta} X-Q X, X) d v_{g}
$$

for $X \in \mathfrak{X}(M)$. Since $J_{I d} V=\bar{\Delta} V-Q V$, we get

$$
g\left(J_{I d} V, V\right)=g(\bar{\Delta} V, V)-g(Q V, V)=\|\nabla V\|^{2}-2 m k .
$$

Then, from (4-3) we have

$$
b \int_{M} g\left(J_{I d} V, V\right) d v_{g}=b \int_{M}\left(\|\nabla V\|^{2}-2 m k\right) d v_{g}=0 .
$$

Smith [1975] proved that if $(M, g)$ is a compact Einstein manifold of dimension $n$, then Id is stable if and only if $\lambda_{1} \geq 2 r / n$, where $\lambda_{1}$ is the first eigenvalue of the Laplace-Beltrami operator acting on functions and $r$ is the scalar curvature. On the other hand, Urakawa [1987, page 572] proved that the first eigenvalue $\lambda_{1}$ of the Laplace-Beltrami operator on $\left(S^{2 m+1} / \Gamma, g\right)$, when $\Gamma \neq\{I d\}$, is bigger than 
or equal to $4 m \kappa$, that is, in such case the identity map $I d$ is stable. Therefore, $g\left(J_{I d} X, X\right) \geq 0$ for any $X \in \mathfrak{X}(M)$. Now take $X=V$ and $b \neq 0$. Then (4-8) gives

$$
\|\nabla V\|^{2}=2 m k=g(Q V, V) .
$$

Moreover, (4-2) becomes $(d+a \kappa)\left(\nabla_{V} V-(\operatorname{div} V) V\right)=0$, from which, since $\nabla_{V} V \perp V$ and $(d+a \kappa) \neq 0$, we have

$$
\nabla_{V} V=0 \quad \text { and } \quad \operatorname{div} V=0 .
$$

From (4-9) and (4-10), applying [Abbassi et al. 2008, Section 6, Proposition 1], we deduce that $V$ is Killing.

We now prove Theorem 1.4(ii). Since $I d$ is stable, from (4-7) we get

$$
(\operatorname{Hess} E)_{I d}(X, X)=\int_{M}\left(\|\nabla X\|^{2}-g(Q X, X)\right) d v_{g} \geq 0
$$

for all $X \in \mathfrak{X}(M)$. Then, applying (2-7) and (4-11), the energy $E_{\widetilde{G}}: \mathfrak{X}^{1}(M) \rightarrow \mathbb{R}$ satisfies

$$
\begin{aligned}
E_{\widetilde{G}}(V) & =\frac{1}{2}((2 m+1)(a+c)+d) \operatorname{vol}(M, g)+\frac{a}{2} \int_{M}\|\nabla V\|^{2} d v_{g} \\
& \geq \frac{1}{2}((2 m+1)(a+c)+d+2 m a \kappa) \operatorname{vol}(M, g)
\end{aligned}
$$

for all $V \in \mathfrak{X}^{1}(M)$. Let $\xi$ be a solenoidal unit vector field (that is, $\operatorname{div} \xi=0$ ). If $\xi:(M, g) \rightarrow\left(T_{1} M, \widetilde{G}\right)$ is a harmonic map, then $\xi$ is Killing by Theorem 1.4(i), and hence $\|\nabla \xi\|^{2}=g(Q \xi, \xi)=2 m \kappa$; see for example [Poor 1981, page 169]. Then, by (4-12), we have $E_{\widetilde{G}}(V) \geq E_{\widetilde{G}}(\xi)$ for all $V \in \mathfrak{X}^{1}(M)$. Vice versa, let $\xi$ be a unit vector field that minimizes the energy, that is,

$$
E_{\widetilde{G}}(\xi)=\frac{1}{2}((2 m+1)(a+c)+d+2 m a \kappa) \operatorname{vol}(M, g) .
$$

Then, by (4-12), $\int_{M}\|\nabla \xi\|^{2} d v_{g}=2 m \kappa \operatorname{vol}(M, g)$, and thus, by (4-11), we get $(\text { Hess } E)_{I d}(\xi, \xi)=0$. Since $I d$ is stable, we can expand $\xi$ into the infinite sum $\xi=\sum_{i=1}^{\infty} E_{i}$ with $J_{I d} E_{i}=a_{i} E_{i}$ and $\int_{M} g\left(E_{i}, E_{j}\right) d v_{g}=0$ for all $i \neq j$, where $a_{i} \geq 0$. Then we have $J_{I d} \xi=\sum_{i=p+1}^{\infty} a_{i} E_{i}$, where $p=\operatorname{dim} \operatorname{ker} J_{I d}$ and $a_{i}>0$ for all $i \geq p+1$. Thus

$$
0=(\operatorname{Hess} E)_{I d}(\xi, \xi)=\int_{M} g\left(J_{I d} \xi, \xi\right) d v_{g}=\sum_{i=p+1}^{\infty} a_{i} \int_{M} g\left(E_{i}, E_{i}\right) d v_{g}
$$

implies that $E_{i}=0$ for any $i \geq p+1$. So, $J_{I d} \xi=0$, that is, $\bar{\Delta} \xi=Q \xi$. Moreover, $\xi$ is a solenoidal unit vector field, that is, $\operatorname{div} \xi=0$; then it is easy to get that $\xi$ is a Killing vector field (see for example [Poor 1981, page 171]), and hence, by Theorem 1.4(i), $\xi:(M, g) \rightarrow\left(T^{1} M ; \widetilde{G}\right)$ is a harmonic map. 


\section{A remark about the energy of unit vector fields}

In Section 4 we characterized the harmonicity of a unit vector field using the energy restricted to $\mathfrak{X}^{1}(M)$. About this energy, Brito [2000] proved the following.

Theorem 5.1. The unit vector fields of minimum energy (with respect to the Sasaki metric) on the unit sphere $S^{3}$ are precisely the unit Killing vector fields.

Brito proved the uniqueness part of his theorem by applying the uniqueness part of Gluck and Ziller's theorem [1986]. On the other hand, the unit sphere $S^{3}$ is a Sasakian three-manifold with constant Webster scalar curvature $w=1$. By a direct method, Perrone [2008] proved the following generalization of Brito's theorem:

Theorem 5.2. Let $(M, g, \xi, \eta)$ be a compact Sasakian three-manifold with Webster scalar curvature $w \geq 1$. Then, the Reeb vector field $\xi$ minimizes the energy, $E_{\widetilde{G}_{s}}(\xi)=\frac{5}{2} \operatorname{vol}(M)$, and the unit vector fields of minimum energy are precisely the unit Killing vector fields $V$ that are eigenvectors of the Ricci operator with eigenvalue 2.

The Ricci operator of a compact Sasakian three-manifold is given by (see for example [Blair 2002, page 105 and 171])

$$
Q=2(2 w-1) I+4(1-w) \eta \otimes \xi .
$$

Thus, $V$ is a unit vector field eigenvector of the Ricci operator with eigenvalue 2 if and only if $(1-w) V=(1-w) \eta(V) \xi$. Then Theorem 5.2 has a direct consequence:

Corollary 5.3. Let $(M, g, \xi, \eta)$ be a compact Sasakian three-manifold. If the Webster scalar curvature $w$ is greater than 1 , the Reeb vector field $\xi$ is, up to sign, the only minimizer of the energy.

Regarding the Webster scalar curvature, the main result of Chern and Hamilton [1985] says that a compact contact three-manifold $(M, \eta)$ admits a contact metric $g$ whose Webster scalar curvature $w$ is either greater than 0 or is a nonpositive constant. Now, let $(M, g, \xi, \eta)$ be a compact Sasakian three-manifold with Webster scalar curvature $w>0$. Consider the $D$-homothetic deformation

$$
g_{t}=t g+\left(t^{2}-t\right) \eta \otimes \eta, \quad \eta_{t}=t \eta, \quad \xi_{t}=(1 / t) \xi,
$$

where $0<t \leq c_{0}=\inf \{w(p): p \in M\}>0$. Then $\left(g_{t}, \eta_{t}, \xi_{t}\right)$ is also a Sasakian structure with Webster scalar curvature $w_{t}$ given by (see [Blair 2002, page 173])

$$
w_{t}=(1 / t) w \geq(1 / t) c_{0} \geq 1 \text {. }
$$

Then $\left(M, g_{t}, \eta_{t}, \xi_{t}\right)$ is a compact Sasakian three-manifold satisfying the condition of Theorem 5.2. In the special case of a compact Sasakian three-manifold 
$(M, g, \xi, \eta)$ with constant Webster scalar curvature $w>0$, the universal covering $\widetilde{M}$ is homothetic to a Berger's sphere. In fact, $\widetilde{M}$ is the Sasakian manifold $\left(S^{3}, g=g_{t}, \xi=\xi_{t}, \eta=\eta_{t}\right)$, where the structure $\left(g_{t}, \xi_{t}, \eta_{t}\right)$ is obtained from the standard Sasakian structure $\left(g_{0}, \xi_{0}, \eta_{0}\right)$ on $S^{3}$ by a $D$-homothetic deformation with $t=1 / w>0$ [Blair 2002]. Since $\eta_{0}=g_{0}\left(\xi_{0}, \cdot\right)$, the metric

$$
\bar{g}_{t}=(1 / t) g_{t}=g_{0}+(t-1) \eta_{0} \otimes \eta_{0}
$$

satisfies

$$
\left.\bar{g}_{t}\right|_{\xi_{0}^{\perp}}=\left.g_{0}\right|_{\xi_{0}^{\perp}}, \quad \bar{g}_{t}\left(\xi_{0}, \xi_{0}\right)=t g_{0}\left(\xi_{0}, \xi_{0}\right), \quad \bar{g}_{t}\left(\xi_{0}, X\right)=0 \quad \text { for } X \in \xi_{0}^{\perp},
$$

where $\xi_{0}^{\perp}$ denotes the orthogonal with respect to the metric $g_{0}$. The Riemannian manifold $\left(S^{3}, \bar{g}_{t}\right)$ is known as a Berger's sphere [Besse 1987, page 252], and $\bar{\xi}_{t}=\sqrt{t} \xi=(1 / \sqrt{t}) \xi_{0}$ is also called a Hopf vector field [Gil-Medrano and Hurtado 2005]. But under the homothetic transformation $\bar{g}_{t}=(1 / t) g_{t}=(1 / t) g$, the energy behaves as follows: if $\bar{V}=\sqrt{t} V$ and $V \in \mathfrak{X}^{1}(M, g)$, then

$$
\begin{aligned}
E_{\widetilde{G}_{s}}\left(\bar{V}, \bar{g}_{t}\right) & =(1 / \sqrt{t})\left(\frac{3}{2}\left(t^{-1}-1\right) \operatorname{vol}(\mathrm{M}, \mathrm{g})\right)+(1 / \sqrt{t}) E_{\widetilde{G}_{s}}(V, g) \\
& \geq(1 / \sqrt{t})\left(\frac{3}{2}\left(t^{-1}-1\right) \operatorname{vol}(M, g)\right)+(1 / \sqrt{t}) E_{\widetilde{G}_{s}}(\xi, g)=E_{\widetilde{G}_{s}}\left(\bar{\xi}_{t}, \bar{g}_{t}\right) .
\end{aligned}
$$

Hence Theorem 5.2 includes the special case of Hopf vector fields $\bar{\xi} g_{t}=(1 / \sqrt{t}) \xi_{0}$ of the Berger's spheres $\left(S^{3}, \bar{g}_{t}\right)$; this special case was first studied in [Gil-Medrano and Hurtado 2005].

We now consider the general compact Sasakian three-manifold $(M, g, \xi, \eta)$ with Webster scalar curvature $w \geq 1$. Then, by Theorem 5.2 and (2-7), we have for any $V \in \mathfrak{X}^{1}(M)$ that

$$
\begin{aligned}
E_{\widetilde{G}}(V) & =\frac{1}{2}[3 c+d] \operatorname{vol}(M, g)+a E_{\widetilde{G}_{s}}(V) \\
& \geq \frac{1}{2}[3 c+d] \operatorname{vol}(M, g)+a E_{\widetilde{G}_{s}}(\xi)=E_{\widetilde{G}}(\xi),
\end{aligned}
$$

that is, $E_{\widetilde{G}}(V) \geq E_{\widetilde{G}}(\xi)$, where the equality holds if and only if $V$ is Killing and $Q V=2 V$. Therefore we get the following:

Theorems 5.1 and 5.2 and Corollary 5.3 are invariant under a four-parameter deformation of the Sasaki metric $\widetilde{G}_{s}$ on $T_{1} M$.

\section{References}

[Abbassi and Calvaruso 2007] K. M. T. Abbassi and G. Calvaruso, " $g$-natural contact metrics on unit tangent sphere bundles”, Monatsh. Math. 151:2 (2007), 89-109. MR 2322938 Zbl 1128.53049

[Abbassi and Sarih 2005] M. T. K. Abbassi and M. Sarih, "On some hereditary properties of Riemannian $g$-natural metrics on tangent bundles of Riemannian manifolds", Differential Geom. Appl. 22:1 (2005), 19-47. MR 2005k:53051 Zbl 1068.53016

[Abbassi et al. 2007] K. M. T. Abbassi, G. Calvaruso, and D. Perrone, "Harmonic sections of tangent bundles equipped with Riemannian $g$-natural metrics", preprint, 2007. arXiv 0710.3668v1 
[Abbassi et al. 2008] K. M. T. Abbassi, G. Calvaruso, and D. Perrone, "Harmonicity of unit vector fields with respect to Riemannian natural metrics", Diff. Geom. Appl. (2008).

[Benyounes et al. 2007a] M. Benyounes, L. E., and C. M. Wood, "Harmonic maps and sections on spheres", preprint, 2007. arXiv math.DG/0703060v1

[Benyounes et al. 2007b] M. Benyounes, E. Loubeau, and C. M. Wood, "Harmonic sections of Riemannian vector bundles, and metrics of Cheeger-Gromoll type", Differential Geom. Appl. 25:3 (2007), 322-334. MR 2008e:53118 Zbl 1128.53037

[Besse 1987] A. L. Besse, Einstein manifolds, Ergebnisse der Mathematik und ihrer Grenzgebiete (3) 10, Springer, Berlin, 1987. MR 88f:53087 Zbl 0613.53001

[Blair 2002] D. E. Blair, Riemannian geometry of contact and symplectic manifolds, Progress in Mathematics 203, Birkhäuser, Boston, 2002. MR 2002m:53120 Zbl 1011.53001

[Brito 2000] F. G. B. Brito, "Total bending of flows with mean curvature correction", Differential Geom. Appl. 12:2 (2000), 157-163. MR 2001g:53065 Zbl 0995.53023

[Brito and Salvai 2004] F. Brito and M. Salvai, "Solenoidal unit vector fields with minimum energy", Osaka J. Math. 41:3 (2004), 533-544. MR 2005h:53044 Zbl 1076.53078

[Chern and Hamilton 1985] S. S. Chern and R. S. Hamilton, "On Riemannian metrics adapted to three-dimensional contact manifolds", pp. 279-308 in Workshop Bonn 1984, edited by F. Hirzebruch et al., Lecture Notes in Math. 1111, Springer, Berlin, 1985. MR 87b:53060 Zbl 0561.53039

[Eells and Sampson 1964] J. Eells, Jr. and J. H. Sampson, "Harmonic mappings of Riemannian manifolds", Amer. J. Math. 86 (1964), 109-160. MR 29 \#1603 Zbl 0122.40102

[Escobales 1975] R. H. Escobales, Jr., "Riemannian submersions with totally geodesic fibers", $J$. Differential Geom. 10 (1975), 253-276. MR 51 \#6650 Zbl 0301.53024

[Gil-Medrano and Hurtado 2005] O. Gil-Medrano and A. Hurtado, "Volume, energy and generalized energy of unit vector fields on Berger spheres: Stability of Hopf vector fields", Proc. Roy. Soc. Edinburgh Sect. A 135:4 (2005), 789-813. MR 2006f:53041 Zbl 1122.53037

[Gluck and Ziller 1986] H. Gluck and W. Ziller, "On the volume of a unit vector field on the threesphere”, Comment. Math. Helv. 61:2 (1986), 177-192. MR 87j:53063 Zbl 0605.53022

[Han and Yim 1998] D.-S. Han and J.-W. Yim, "Unit vector fields on spheres, which are harmonic maps", Math. Z. 227:1 (1998), 83-92. MR 99c:58044 Zbl 0891.53024

[Ishihara 1979] T. Ishihara, "Harmonic sections of tangent bundles", J. Math. Tokushima Univ. 13 (1979), 23-27. MR 81b:58015 Zbl 0427.53019

[Koláŕ et al. 1993] I. Kolář, P. W. Michor, and J. Slovák, Natural operations in differential geometry, Springer, Berlin, 1993. MR 94a:58004 Zbl 0782.53013

[Nouhaud 1977] O. Nouhaud, "Applications harmoniques d'une variété riemannienne dans son fibré tangent: Généralisation”, C. R. Acad. Sci. Paris Sér. A-B 284:14 (1977), A815-A818. MR 55 \#4037 Zbl 0349.53015

[Perrone 1998] D. Perrone, "Homogeneous contact Riemannian three-manifolds", Illinois J. Math. 42:2 (1998), 243-256. MR 99a:53067 Zbl 0906.53031

[Perrone 2003] D. Perrone, "Harmonic characteristic vector fields on contact metric three-manifolds”, Bull. Austral. Math. Soc. 67:2 (2003), 305-315. MR 2004c:53097 Zbl 1034.53050

[Perrone 2005] D. Perrone, "The rough Laplacian and harmonicity of Hopf vector fields", Ann. Global Anal. Geom. 28:1 (2005), 91-106. MR 2006d:53064

[Perrone 2008] D. Perrone, "On the volume of unit vector fields on Riemannian three-manifolds", 2008. To appear in C. R. Acad. Sc. Canada. 
[Poor 1981] W. A. Poor, Differential geometric structures, McGraw-Hill Book Co., New York, 1981. MR 83k:53002 Zbl 0493.53027

[Rukimbira 2002] P. Rukimbira, "Criticality of $K$-contact vector fields", J. Geom. Phys. 40:3-4 (2002), 209-214. MR 2002k:53153 Zbl 1007.53048

[Smith 1975] R. T. Smith, "The second variation formula for harmonic mappings", Proc. Amer. Math. Soc. 47 (1975), 229-236. MR 51 \#11580 Zbl 0303.58008

[Tsukada and Vanhecke 2001] K. Tsukada and L. Vanhecke, "Minimality and harmonicity for Hopf vector fields”, Illinois J. Math. 45:2 (2001), 441-451. MR 2003i:53097 Zbl 0997.53040

[Urakawa 1987] H. Urakawa, "Stability of harmonic maps and eigenvalues of the Laplacian", Trans. Amer. Math. Soc. 301:2 (1987), 557-589. MR 88g:58046 Zbl 0621.58010

[Wiegmink 1995] G. Wiegmink, "Total bending of vector fields on Riemannian manifolds", Math. Ann. 303:2 (1995), 325-344. MR 97a:53050 Zbl 0834.53034

Received June 11, 2008.

DOMENICO PERRONE

Dipartimento di Matematica "Ennio De Giorgi”

UNIVERSITÀ DEL SALENTO

C.P. 193

Provinciale LeCCE-ARnesano

73100 LECCE

ITALY

domenico.perrone@unile.it 